\title{
Compressive Strain Behaviour under Different Strain Rates in Multi- Walled Carbon Nanotubes-Polycarbonate Composites
}

\section{Prashant Jindal}

University Institute of Engineering and Technology, Panjab University, Chandigarh 160014, India

\begin{abstract}
Polycarbonate $(\mathrm{PC})$ based composites were fabricated with different concentrations of Multi Walled Carbon Nanotubes (MWCNTs) and were subjected to dynamic impact loading using Split Hopkinson Pressure Bar (SHPB). Impact tests using this instrument were performed under varying strain rates ranging from 1096 to 4017/s on pure PC samples and samples with $0.1,0.5$ and $1 \%$ by weight of MWCNT concentrations in PC. A comparison of maximum strains produced in these samples due to different strain rates was done. It was found that the maximum strain produced for every sample increases with increase in strain rate. Maximum strain is a measure of the toughness and deformation of the sample which increases as strain rate increases.
\end{abstract}

Keywords: Strain rate; Maximum strain; Polycarbonate; Carbon nanotubes

\section{Introduction}

Stress behavior of materials at high strain rates is a very important parameter for shock and energy absorbing related applications. However, along with stress, it is very important to see how much deformation the sample undergoes when it experiences heavy impact loads. The maximum strain that a material undergoes gives a measure of the energy required for the material to undergo permanent fracture, in other words it gives the measure of its toughness. For any practical shock load related application, the specimen which undergoes such heavy impact must be capable of absorbing the load and its deformation also becomes important to analyse its repeated use. Shielding specimen can be one time use substances or can be used multiple times also depending upon their state after first time use. However, dynamic impact loads generally cause a permanent strain or deformation in the specimen. Moreover, various composites exhibiting properties to absorb heavy impact loads must also be studied for the maximum strain they undergo before failing because if the space where that specimen is installed does not permit this deformation, then it can escalate the damage to other parts of the host machinery. So, it becomes imperative to evaluate the dimensional change caused in samples.

Over the past few decades, materials have exhibited similar behavior of increased strain when they undergo higher strain rates. Ever since the synthesis of Carbon Nanotubes (CNTs) [1] and study that followed exploring mechanical and structural properties of CNTs [2-4], there has been wide ranging interests in scientific and engineering communities to exploit these for varying applications. The unusual mechanical strength of the carbon nanotubes revealing them as about 100 times stronger than steel motivates to fabricate and modify useful materials which are cheaply available in bulk form by embedding in these carbon nanotubes in various forms to make composites which have desired mechanical properties. For low strain rate range, Weeks et al. [5], has shown that as strain rate increases from $10 \mu$ /s to 100,000 $\mu / s$ on AS4 thermoplastics off axis composites the strain behavior shows a non-linear behavior. The maximum strain varies from $1.7 \%$ to $2.7 \%$ in the above strain range. Renliang et al. [6], used the specimen as granite and marble and performed impact test using SHPB. It was found that stress strain behavior was linear for a small strain range and beyond that a non-linear pattern was reported. The high strain rate has been measured as high impact velocity and as velocity is increasing the maximum strain is also increasing from $100 \times 10^{-4} \%$ to $250 \times 10^{-4} \%$. Another elastic material which was tested using SHPB by Ramezani et al. [7]. It showed the stress strain pattern for strain rates of 180/s, 230/s, $300 / \mathrm{s}$ and 350/s. The maximum stress for this specimen kept increasing with increase in strain rate and maximum strain also increase from $0.6 \%$ to $0.8 \%$. Tension test was performed on polycarbonate for strain rates in the range of 370/s to 1700/s by Kan Cao et al. [8], and again an increase of maximum strain was reported. Siviour et al. [9], performed compression impact tests on polycarbonates and polyvinylidene diflouride for strain rates of nearly $10^{4} / \mathrm{s}$ at different temperatures. Maximum strains for these samples also increased with strain rate. Chen et al. [10], performed similar tension and compression tests on PMMA and Epon 828/T-403 using SHPB. The results reported do not show much variation on strain due to variation in strain rates which are in the order of $1000 / \mathrm{s}$ to $5000 / \mathrm{s}$.

In an earlier study, we had fabricated PC-MWCNT composite samples and studied their dynamic impact resistance behavior using SHPB [11]. From the same experiments, we hereby study the effect on concentration on the strain produced due to dynamic impact loading.

\section{Results and Discussion}

The impact loading was performed for various strain rates. Strain rates in the range $2000 / \mathrm{s}$ to $2600 / \mathrm{s}$ is applicable in defence related applications [12], so the performance of samples within this range needs to analysed. Maximum and elastic limit strains which the samples exhibited in the range of low strain rates (nearly 1500/s) and high strain rates (nearly 3000/s) have been depicted in table 1 . It shows that both elastic limit strain and maximum strain increase for every sample irrespective of the sample concentration as strain rate increases.

*Corresponding author: Prashant Jindal, University Institute of Engineering and Technology, Panjab University, Chandigarh 160014, India, E-mail: jindalp@pu.ac.in

Received December 04, 2012; Accepted December 07, 2012; Published December 15, 2012

Citation: Jindal P (2013) Compressive Strain Behaviour under Different Strain Rates in Multi-Walled Carbon Nanotubes-Polycarbonate Composites. J Material Sci Eng 2:119. doi:10.4172/2169-0022.1000119

Copyright: (c) 2013 Jindal P. This is an open-access article distributed under the terms of the Creative Commons Attribution License, which permits unrestricted use, distribution, and reproduction in any medium, provided the original author and source are credited. 
Citation: Jindal P (2013) Compressive Strain Behaviour under Different Strain Rates in Multi-Walled Carbon Nanotubes-Polycarbonate Composites. J Material Sci Eng 2:119. doi:10.4172/2169-0022.1000119

Page 2 of 3

\begin{tabular}{|l|c|c|}
\hline Strain(s-1) & Elastic limit strain(\%) & Maximum strain(\%) \\
\hline For Pure PC sample & 8 & 45 \\
\hline 1576 & 10 & 112 \\
\hline 2778 & 10 & 112 \\
\hline 3300 & 16 & 229 \\
\hline 3900 & 10 & 99 \\
\hline For 0.1\% MWCNT-PC composite & 10 & 108 \\
\hline 2609 & 12 & 144 \\
\hline 2778 & 8 & 66 \\
\hline 3200 & 10 & 73 \\
\hline For 0.5\% MWCNT-PC composite & 10 & 108 \\
\hline 2032 & 10 & 113 \\
\hline 2186 & & \\
\hline 2768 & 9 & 53 \\
\hline 2845 & 10 & 92 \\
\hline For 1.0\% MWCNT-PC composite & 10 & 116 \\
\hline 1643 & 12 & 136 \\
\hline 2547 & & \\
\hline 2926 & & \\
\hline 3133 & & \\
\hline
\end{tabular}

Table 1: Variation of elastic limit strain and maximum strain for different compositions at various strain rates.

Previous paper studies indicated that major increase in stress resistance for pure PC takes place for samples with $0.5 \%$ MWCNTs in PC. Figures 1-3 indicate the stress-strain results for pure PC, $0.1 \%$ MWCNT-PC and 0.5\% MWCNT-PC under different strain rates graphically. The maximum stress for each composition does not show any uniform variation with strain rate for every composition. However, for our present study, since deformation (strain) is the main focus we observe that as strain rate increases the maximum strain for every composition increases before being crushed. Considering, that if any equipment is complex with several small important devices attached in a very limited space arrangement and it gets exposed to heavy dynamic loading then the impact absorbing or protective shield has to not only absorb the heavy impact but also assure that its deformation does not disturb the other small devices. So, owing to space limitations such studies become imperative.

From the figures 1-3, it becomes evident that PC based samples can deform to a large extent as the dynamic impact load increases which is a similar behavior to various other materials like AS4 thermoplastics, granite, marble etc [5-8].

Figure 4 indicates the strain behavior for the four main concentrations of pure PC, $0.1 \%$ MWCNT-PC and 0.5\% MWCNT-PC. Addition of small quantities of MWCNTs in PC enhances the stress [11] but it does not increase the maximum strain. This is evident from figure 4 as the samples with or without MWCNTs does not show much strain variation at high strain rates. These materials after crossing the elastic limit tend to lose their strength and as a result start deforming very rapidly even if the applied external load is reduced. Therefore, during dynamic loading maximum stress and maximum strain become important points. Although, MWCNTs do not enhance the maximum strain but their embedding in PC enhances the maximum stress due to which their application for such applications becomes critical.

\section{Summary and Conclusion}

This paper highlights the role of strain rate on the stress-strain behavior of MWCNT-PC composites with specific reference to maximum strain of the specimen. In an earlier paper, mechanical properties at a given strain rate of these composites were presented.
Whether the pure PC is embedded with MWCNTs or not, the maximum strain increases with increase in strain rate.

Small quantities of MWCNTs when embedded in pure PC enhance its impact absorption capacity by nearly 10-15\% [11]. Apart from this, the deformation that takes place when these specimens are subjected

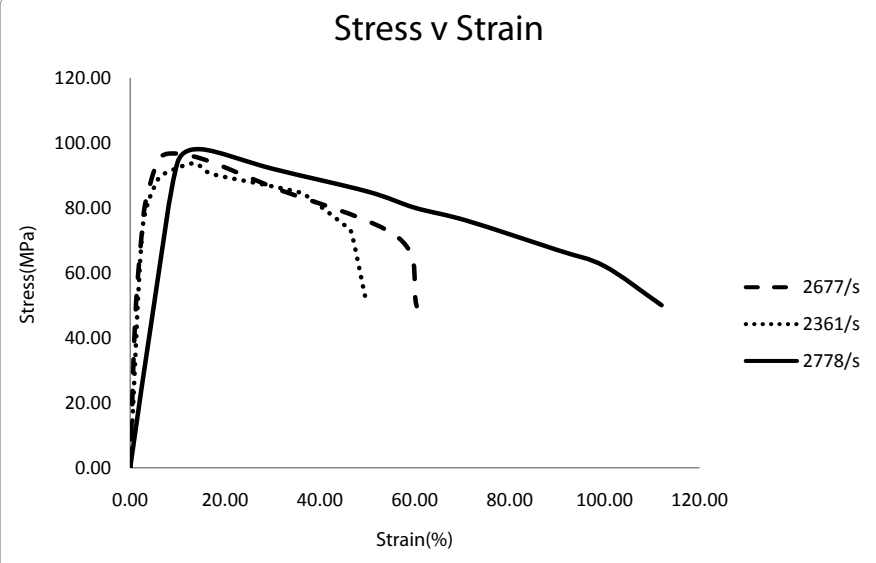

Figure 1: Stress-Strain variation for pure PC under different strain rates.

Stress v Strain

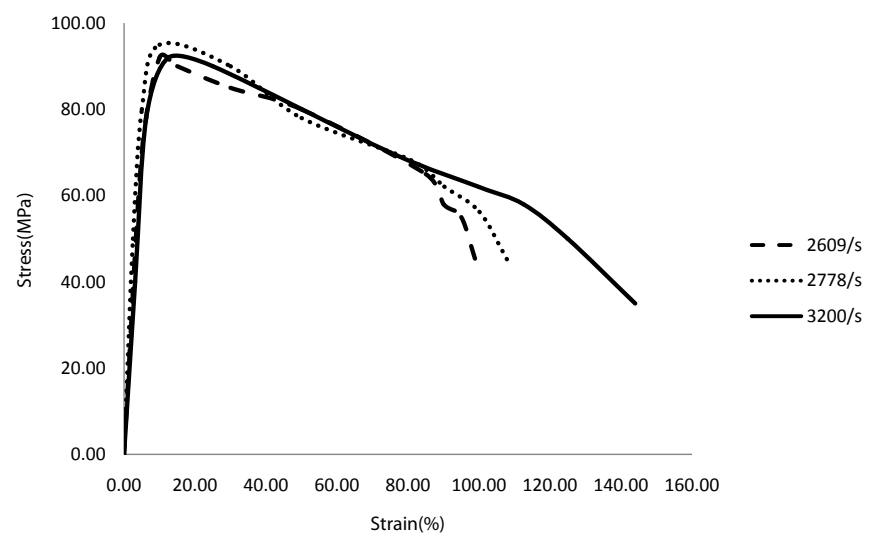

Figure 2: Stress-Strain variation for $0.1 \%$ MWCNT-PC under different strain rates.

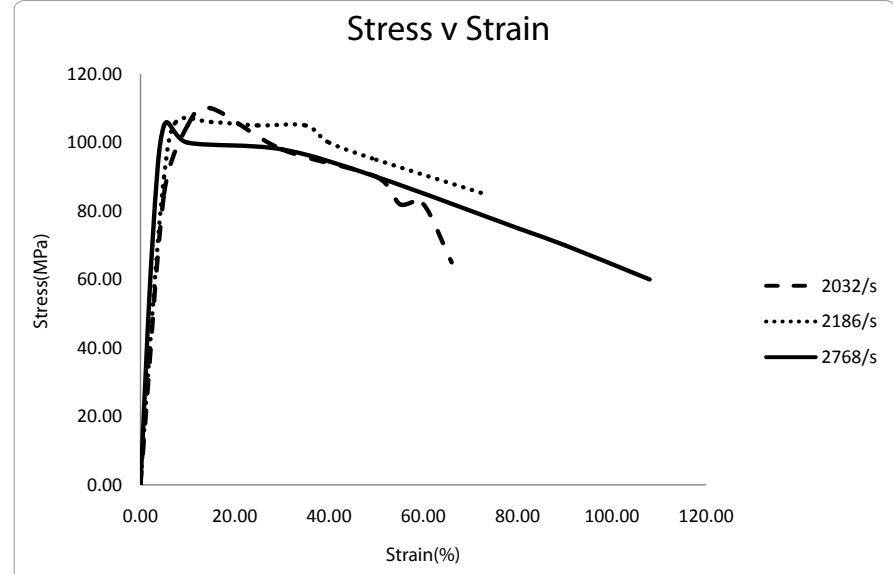

Figure 3: Stress-Strain variation for $0.5 \%$ MWCNT-PC under different strain rates. 
Citation: Jindal P (2013) Compressive Strain Behaviour under Different Strain Rates in Multi-Walled Carbon Nanotubes-Polycarbonate Composites. J Material Sci Eng 2:119. doi:10.4172/2169-0022.1000119

Page 3 of 3

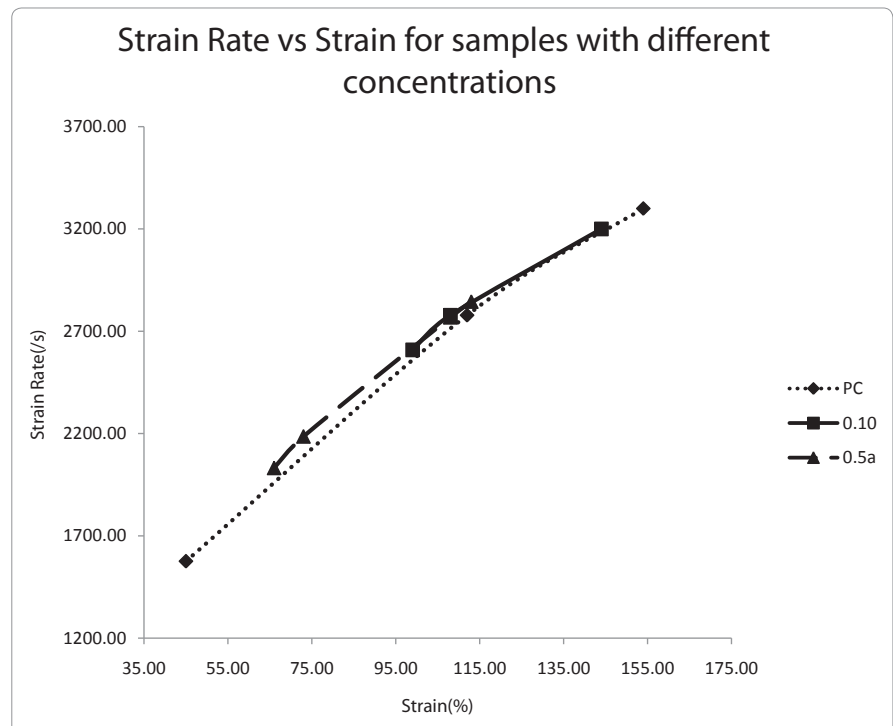

Figure 4: Variation of strain rate with strain for pure PC, $0.1 \%$ MWCNT-PC and $0.5 \%$ MWCNT-PC.

to such high impact loads also becomes a matter of concern. When the impact load process is over, this specimen deforms and in the final state may get even broken or crushed. However, during deformation it dimensional change may cause interference to various other devices installed around, which can escalate the damage caused by the impact. So, the specimen that is installed for protection from heavy impact load must conform to the space around it and its deformation must also be ascertained. More samples and data is required to see when the strain starts decreasing or remains unchanged with increase in strain rate, as the limit has to be explored.

\section{Acknowledgements}

Prashant Jindal gratefully acknowledges financial support from the
Defence Research Organization (DRDO) for a research project (No: ARMREB/ DSW/2011/129). He also acknowledges the Director TBRL and Gun Group, TBRL for extending facilities at his lab. Author is also grateful to the National Physical Laboratory, New Delhi for their assistance in sample fabrication.

\section{References}

1. Endo M, lijima S, Dresselhaus MS (1996) Carbon Nanotubes. 33

2. Ruoff RS, Qian D, Liu WK (2003) Mechanical properties of carbon nanotubes: theoretical predictions and experimental measurements. Comptes Rendus Physique 4: 993-1008.

3. Sears A, Batra RC (2004) Macroscopic properties of carbon nanotubes from molecular-mechanics simulations. Phys Rev B 69: 235406.

4. Dresselhaus MS, Dresselhaus G, Eklund PC (1996) Science of Fullerenes and Carbon Nanotubes: Their Properties and Applications. Acad Press, USA.

5. Weeks CA, Sun CT (1998) Modeling non-linear rate-dependent behavior in fiber-reinforced composites. Compos Sci Technol 58: 603-611.

6. Shan R, Jiang Y, Li B (2000) Obtaining dynamic complete stress-strain curves for rock using the Split Hopkinson Pressure Bar technique. Int J Rock Mech Min 37: 983-992.

7. Ramezani M, Ripin ZM (2010) Combined experimental and numerical analysis of bulge test at high strain rates using split Hopkinson pressure bar apparatus. J Mater Process Tech 210: 1061-1069.

8. Cao K, Ma X, Zhang B, Wang Y, Wang YU (2010) Tensile behavior of polycarbonate over a wide range of strain rates. Mat Sci Eng; A 527: 40564061.

9. Siviour CR, Walley SM, Proud WG, Field JE (2005) The high strain rate compressive behaviour of polycarbonate and polyvinylidene difluoride. Polymer 46: $12546-12555$.

10. Chen W, Lu F, Cheng M (2002) Tension and compression tests of two polymers under quasi-static and dynamic loading. Polym Test 21: 113-121.

11. Jindal P, Pande S, Sharma P, Mangla V, Chaudhury A, et al. (2013) High strain rate behavior of multi-walled carbon nanotubes-polycarbonate composites. Compos Part B-Eng 45: 417-422.

12. Mostafa S, David N, Vikas P (2003) Modeling of high-strain-rate deformation fracture, and impact behavior of advanced gas turbine engine materials at low and elevated temperatures. National Aeronautics and Space Administration, Glenn Research Center. 